\title{
Identification and Anti-Fungal Resistance Profile of Different Candida Species Isolated from Patients in ICUs
}

\author{
Ghada E. Amr1, Dina M. Atef ${ }^{1^{*}}$ and Ahmed M. Salah ${ }^{2}$ \\ ${ }^{1}$ Clinical and Chemical Pathology Department, Faculty of Medicine, \\ Zagazig University, Egypt \\ ${ }^{2}$ Internal Medicine Department, Faculty of Medicine, Zagazig University, Egypt \\ *Corresponding author
}

\begin{abstract}
A B S T R A C T
Candidiasis is one of the most predominant fungal infections that cause a major mortality

\section{Keywords}

Candida, Candida non albicans, Antifungal susceptibility, MSMALDI TOF

Article Info

Accepted:

07 May 2019

Available Online:

10 June 2019

of the patient. The epidemiology of candida infections have been witnessed a change round the world. We aimed to determine the distribution of candida species isolates from various clinical samples of patients in ICUs and evaluate its antifungal susceptibility to demonstrate the local resistance profile and guiding empirical treatment for clinicians. This study included 714 Candida isolates. Candida species were identified by MALDI-TOF mass spectrometry. Antifungal susceptibility tests were performed by Vitek 2 system. The percentage of NAC isolates $(63.59 \%)$ was higher compared with C. albicans $(36.41 \%)$. The highest resistance rate in candida species was against amphotericin B (11.56\%). However there was no resistance to caspofungin and micafungin reported. Regarding to voriconazole and fluconazoleanti fungal, the resistance rate of $C$. albicans were $13.46 \%$ and $8.46 \%$ respectively while of NAC species were $6.53 \%, 5.1 \%$ respectively. All $C$. krusei isolates were resistance to fluconazole. The study revealed increased the incidence of NAC over time. Although no remarkable resistance for antifungal agents in our hospital, we establish a critical need to antifungal stewardship program to prevent reaching the era of predominant Multi- drug resistance strain of Candida.
\end{abstract}

\section{Introduction}

In the past few decades, the incidence of mycotic infection has trend to increase progressively throughout the world (Pfaller 2007). Candida infection is diverse, including asymptomatic colonization, cutaneous candidiasis, or pharyngeal candidiasis and invasive candidiasis including candidemia (Anwar et al., 2012). Nosocomial candidiasis is among the most predominant fungal infection that causes a major hazard on the health of patients from centers of tertiary care and spread to the community hospitals (Singhi and Deep, 2009). In the United States, $5 \%-10 \%$ of patients admitted in hospitals had acquired nosocomial infection. Candida caused about $80 \%$ of fungal infections. Several studies observed that Candida albicans (C. albicans) is the sixth commonest cause of nosocomial infections (Pappas et al., 2009). 
Recently, $C$. albicans has been the most common species implicated, when the incidence of non-albicans Candida (NAC) has risen dramatically (Bajwa and Kulshrestha, 2013). With previous study, the epidemiology of Candida infections have been witnessed a change in many countries round the world, characterized by a progressive shift from a predominance of $C$. albicans to NAC species (Oberoi et al., 2012).

The frequency of Candida species other than C. albicans varies according to various geographical regions and over time (Pfaller 2007). For example, Candida glabrata is the second common species after $C$. albicans in North America (Pfaller and Diekema, 2012). However, in Asia, Australia and Europe, C.tropicalis and C. parapsilosis are more common (Chen et al., 2006 and Wang et al., 2010). C. tropicalis is main cause of candidaemia in India (Xiao et al., 2014). Additionally, uncommon species such as $C$. guilliermondii and C. rugosa are emerging (Chander et al., 2013).

A number of virulence factors are the explanation for the alteration of different Candida spp. from commensal to vigorous infectious agent, like biofilm formation, adherence to host tissues and medical devices and secretion of extracellular hydrolytic enzymes (Sardi et al., 2013). Even though many studies that published data about the risk factors that cause increasing the risk of Candida infection in intensive care unit (ICU).Of those risk factors, severe immunosuppression or illness, increased use of invasive procedures and devices, abdominal surgery, frequent use of broad spectrum antibiotics and empirical use of antimycotic drugs are reported to be associated with such an increase (Goemaere et al., 2018 and Trick et al., 2002).

The aim of our study was to determine the distribution of different Candida species isolates from numerous clinical specimens and evaluate its antifungal susceptibility to demonstrate the local resistance profile of ICUs patients in Zagazig University Hospitals and to guide empirical treatment for clinicians.

\section{Materials and Methods}

This is a retrospective study of all Candida isolates data retrieved from the computerized databases of the microbiology unit of Clinical Pathology Department, Zagazig University Hospitals and included 714 Candida species isolated from patients who admitted to ICUs between the period of November 2016 and December 2018. All the investigations were performed following the relevant guidelines and regulations of Zagazig University.

All Patients included in the study were suffered from symptoms and signs of infection; other bacterial causes were excluded by different culture technique. Patients were diagnosed on the basis of clinical presentation; they were subjected to full history taking with focusing on the risk factors association such as hospital stay duration, underlying medical conditions, invasive medical procedures such as presence of urinary catheter, central line insertion, respiratory ventilation and the usage of antibiotics, chemotherapy, corticosteroids or immunosuppressive drugs.

\section{Samples collection}

Non-duplicate isolates were recovered during the study period. Candida species were revealed from numerous clinical samples such as blood, respiratory samples as bronchoalveolar lavage, deep tracheal aspirate, pleural fluid and sputum, urine and others miscellaneous sites as oropharyngealand vaginal swabs. Blood cultures were done using Bact /ALERT culture bottles and incubated for 7 days in the 
automated Bact / ALERT 3D Microbial Detection System (bioMérieux, Inc, Durham, USA). The positive blood culture bottles and other isolated samples were initially grown on blood agar and sabouraud dextrose agar for twenty four to forty eight hours at $37^{\circ} \mathrm{C}$.

\section{Identification}

Candida colonies appeared as flat, smooth and pale off white coloured and identified by gram stain. Germ tube formation had been tested for the isolated colony to differentiate C. albicans against NAC. Candida species were Identified using matrix-assisted laser desorption ionization-time of flight mass spectrometry (MALDI-TOF MS) (bioMérieux, Marcy l'Etoile, France) according to the manufacturer instructions. Small amount of the freshly Candida colonies were picked and smeared on the wells of disposable target slides, $1 \mu \mathrm{L}$ formic acid was added and air dried then $1 \mu \mathrm{L}$ of VITEK MS CHCA matrix solution (cyano-4hydroxycinnamic acid) was added to the sample and left to dry at room temperature for 1- $2 \mathrm{~min}$. Then target slide was loaded into the VITEK MS, the mass spectra acquired for each sample were compared to the known mass spectra in the database and given a confidence score.

\section{Antifungal susceptibility testing}

The susceptibility to antifungal agents was carried out using Vitek 2 system (bioMérieux, Inc, Durham, USA)for yeast (card no ACT/YS07) containing serial twofold dilutions of six antifungal drugs; fluconazole, flucytosine, voriconazole, caspofungin, micafungin and amphotericin $\mathrm{B}$ were provided by the manufacturer and the antifungal susceptibilities were interpreted according to the Clinical and Laboratory Standards Institute guidelines (CLSI)after adjustment of McFarland standard of 2 with colony inoculums. The results were interpreted as sensitive (S), intermediate (I) and resistant $(\mathrm{R})$.

Minimal inhibition concentrations (MICs) cut- off values for flucytosine $\leq 1 \mu \mathrm{g} / \mathrm{ml}$ was considered to be susceptible (S) and $\geq 64$ $\mu \mathrm{g} / \mathrm{ml}$ was considered to be resistant (R), MIC was categorized for fluconazole as $\leq 1 \mu \mathrm{g} / \mathrm{ml}$ (S) and $\geq 64 \mu \mathrm{g} / \mathrm{ml}(\mathrm{R})$, for voriconazole was $\leq 0.12 \mu \mathrm{g} / \mathrm{ml}$ (S) and $\geq 8 \mu \mathrm{g} / \mathrm{ml}$ (R), for amphotericin B was $\leq 0.25 \mu \mathrm{g} / \mathrm{ml}(\mathrm{S})$ and $\geq 16$ $\mu \mathrm{g} / \mathrm{ml}(\mathrm{R})$, for micafungin was $\leq 0.06 \mu \mathrm{g} / \mathrm{ml}$ (S) and $\geq 4 \mu \mathrm{g} / \mathrm{ml}(\mathrm{R})$ and for caspofungin was $\leq 0.25 \mu \mathrm{g} / \mathrm{ml}$ to be (S) while $\geq 4 \mu \mathrm{g} / \mathrm{ml}$ was considered to be (R). The ranges of MICs at which $50 \%$ and $90 \%$ of the isolates population of Candida species had been inhibited $\left(\mathrm{MIC}_{50}\right.$ and $\mathrm{MIC}_{90}$, respectively) were calculated. The acceptable percent essential agreement for MICs was set at $\geq$ $90 \%$ for each antifungal agent against all organisms tested.

\section{Statistical analysis}

The data was statistically analyzed using SPSS statistical package software computer program version 17 (SPSS Inc., Chicago, IL). Categorical variables are presented as number and percentage. The relationship between usage of antifungal and the distribution of Candida species were determined using the Chi-squared test for non-parametric correlation. $\mathrm{P}$ value $<0.05$ was considered significant.

\section{Results and Discussion}

The demographic clinical characteristics by the most common Candida species were shown in table 1: Candida species was seen in 714 isolates from different specimens. NAC $(63.59 \%)$ was isolated from patients admitted in ICU compared to $C$. albicans (36.41\%) during the studied period $(\mathrm{P}<0.0001)$.This result showed anincrease of the incidence of 
NAC from epidemiological data presented in our hospitals covering the periods from January 2014 to November 2016 found that the incidence of NAC species was $(59.4 \%)$. The duration of hospital stay (>1 week) was significantly increased inpatients with NAC compared to these with $C$. albicans $(\mathrm{p}<0.001)$.

The prevalence rate of Candida species isolated from various clinical specimens: As shown in table 2, isolation of different types of Candida species from different clinical specimens showed the majority in urine samples $(61 \%)$, followed by respiratory tract specimens $(16 \%)$, blood samples $(7.9 \%)$ and finally $(15.1 \%)$ isolates from different miscellaneous samples. The prevalence of Candida species was as follows: C. albicans, (36.4\%), C. tropicalis (57.7\%), C. glabrata (2.1\%), C. krusei (1.26), C. parapsilosis $(1.1 \%), \quad$ C. lusitaniae $(0.6 \%), \quad$ C. kefyr $(0.42 \%)$ and $0.14 \%$ for C. dubliniensis, $C$. guilliermondii and $C$. rugosa.

The rate of resistance of Candida isolates to anti-fungal agents was shown in table 3: The highest resistance rate in all Candida species was against amphotericin B (11.56\%). However there was no resistance to caspofungin and micafungin (echinocandins class of antifungal) was reported. Low resistance was showed to flucytosine $(2.4 \%)$ among Candida species.

Table.1 Demographic clinical characteristics by the most common Candida species

\begin{tabular}{|l|c|c|c|}
\hline \multicolumn{1}{|c|}{ Candida SPP. } & C. albicans & C. Non Albicans & P value \\
\hline Number of isolated & $260(36.41 \%)$ & $454(63.59 \%)$ & $<0.0001$ \\
\hline Gender: & $203(78.1 \%)$ & $315(69.4 \%)$ & 0.03 \\
Male & $57(21.9 \%)$ & $139(30.6 \%)$ & 0.21 \\
\hline Female & & & \\
\hline Underlying condition: & $78(30 \%)$ & $142(31.3 \%)$ & 0.84 \\
Diabetes Mellitus & $114(43.8 \%)$ & $191(42.1 \%)$ & 0.77 \\
Heart/ Pulmonary diseases & $39(15 \%)$ & $74(16.3 \%)$ & 0.85 \\
Malignancy & $57(21.9 \%)$ & $88(19.4 \%)$ & 0.71 \\
\hline Renal Diseases & & & \\
\hline Predisposing factors: & $32(12.3 \%)$ & $69(15.2 \%)$ & 0.69 \\
GIT diseases/ operation & $63(24.3 \%)$ & $126(27.7 \%)$ & 0.61 \\
Receipt of Corticosteroids & $45(17.3 \%)$ & $137(30.2 \%)$ & 0.09 \\
Central venous catheter & $170(65.4 \%)$ & $284(62.5 \%)$ & 0.53 \\
Indwelling of urinary catheter & $39(15 \%)$ & $55(12.1 \%)$ & 0.68 \\
Receipt of mechanical ventilation & $89(34.2 \%)$ & $107(23.6 \%)$ & 0.102 \\
Blood product transfusion & $146(56.1 \%)$ & $289(63.7 \%)$ & 0.12 \\
Candiduria & $51(19.6 \%)$ & $112(24.7 \%)$ & 0.47 \\
\hline Antifungal prophylaxis & & & \\
\hline Outcome: & $18(6.9 \%)$ & $46(10.1 \%)$ & 0.69 \\
Mortality (30 days) & $158(60.77 \%)$ & $(372(81.93 \%)$ & $<0.0001$ \\
\hline ICU stays (> 1 wk)
\end{tabular}

Significant if $\mathrm{P}$ value $<0.05$ Data are presented as No. (\%) 
Table.2 The prevalence rate of Candida spp. isolated from various clinical specimens.

\begin{tabular}{|c|c|c|c|c|c|}
\hline $\begin{array}{l}\text { Cl. specimen } \\
\text { Candida spp }\end{array}$ & $\begin{array}{c}\text { Urine } \\
(n=435)\end{array}$ & $\begin{array}{c}\text { Respiratory } \\
(n=115)\end{array}$ & $\begin{array}{l}\text { Blood } \\
(n=57)\end{array}$ & $\begin{array}{c}\text { Miscellaneous } \\
(\mathrm{n}=\mathbf{1 0 8})\end{array}$ & $\begin{array}{c}\text { Total } \\
(n=714)\end{array}$ \\
\hline C. albicans & $146(33.56 \%)$ & $40(34.78 \%)$ & $28(49.1 \%)$ & $46(42.6 \%)$ & $260(36.4 \%)$ \\
\hline C. Non albicans: & $289(66.44 \%)$ & $75(65.22 \%)$ & $29(50.9 \%)$ & $62(57.4 \%)$ & $454(63.6 \%)$ \\
\hline C. tropicalis & $269(61.84 \%)$ & $67(58.3 \%)$ & $29(50.9 \%)$ & $48(44.44 \%)$ & $412(57.7 \%)$ \\
\hline C. glabrata & $6(1.4 \%)$ & $3(2.6 \%)$ & - & $6(5.55 \%)$ & $15(2.1 \%)$ \\
\hline C. krusei & $3(0.7 \%)$ & $3(2.6 \%)$ & - & $3(2.78 \%)$ & $9(1.26 \%)$ \\
\hline C. parapsilosis & $5(1.11 \%)$ & $1(0.86 \%)$ & - & $2(1.85 \%)$ & $8(1.1 \%)$ \\
\hline C. leusitaniae & $1(0.23 \%)$ & $1(0.86 \%)$ & - & $2(1.85 \%)$ & $4(0.6 \%)$ \\
\hline C. kefyr & $3(0.7 \%)$ & - & - & - & $3(0.42 \%)$ \\
\hline C. dubliniensis & $1(0.23 \%)$ & - & - & - & $1(0.14 \%)$ \\
\hline $\begin{array}{l}\text { C. } \\
\text { guilliermondii }\end{array}$ & - & - & - & $1(0.93 \%)$ & $1(0.14 \%)$ \\
\hline C. rugosa & $1(0.23 \%)$ & - & - & - & $1(0.14 \%)$ \\
\hline
\end{tabular}

Data are presented as No. (\%)

Table.3 The resistance rate of Candida species to antifungal agents

\begin{tabular}{|c|c|c|c|c|c|c|c|}
\hline \multirow[b]{2}{*}{ Candida spp. } & \multirow{2}{*}{$\begin{array}{l}\text { No. of } \\
\text { isolates }\end{array}$} & \multicolumn{6}{|c|}{ Antifungal agent } \\
\hline & & Amphotericin B & Voriconazole & Fuconazole & Flucytosine & Caspofungin & Micafungin \\
\hline C. albicans & 260 & $43(16.53 \%)$ & $35(13.46 \%)$ & $22(8.46 \%)$ & $\begin{array}{c}2 \\
(0.77 \%)\end{array}$ & 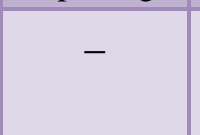 & - \\
\hline C. non albicas & 449 & $39(8.68 \%)$ & $31(6.9 \%)$ & $31(6.9 \%)$ & $\begin{array}{c}15 \\
(3.34 \%)\end{array}$ & - & - \\
\hline C. tropicalis & 412 & $32(7.77 \%)$ & $27(6.53 \%)$ & $21(5.1 \%)$ & $\begin{array}{c}13 \\
(3.15 \%)\end{array}$ & - & - \\
\hline C. glabrata & 15 & $2(13.33 \%)$ & $2(13.33 \%)$ & $1(11.11 \%)$ & $1(6.67 \%)$ & - & - \\
\hline C. parapsilosis & 8 & $2(25 \%)$ & - & - & - & - & - \\
\hline C. krusei & 9 & $2(22.22 \%)$ & $1(11.11 \%)$ & $9(100 \%)$ & $\begin{array}{c}1(11.11 \% \\
)\end{array}$ & - & - \\
\hline C. leusitaniae & 4 & - & $1(25 \%)$ & & & - & - \\
\hline C. rugosa & 1 & $1(100 \%)$ & - & - & - & - & - \\
\hline Total \% of resistance & 709 & $82(11.56 \%)$ & $66(8.46 \%)$ & $53(7.47 \%)$ & $17(2.4 \%)$ & - & - \\
\hline
\end{tabular}

Data are presented as No. (\%) 
Regarding to triazoles antifungal, the resistance rate of $C$. albicans to voriconazole was $13.46 \%$ and $8.46 \%$ to fluconazole. In addition, the resistance rate to voriconazole and fluconazole among NAC species had been reported as $6.53 \%, 5.1 \%$ respectively. All C. krusei isolates were resistance to fluconazole $(100 \%)$.

Candida has emerged as principal cause for fungal infections worldwide, which is responsible of high morbidity and mortality in patients who admitted in hospitals with serious underlying diseases (Colombo et al., 2007).However in recent years there is an increasing prevalence of infections caused by NAC species of Candida compared to $C$. albicans which shows decreased incidence (Wang et al., 2016). Limited epidemiological studies of Candida infection have been done in Egypt and the surrounding countries, in contrast to the extensive numbers of studies done in Europe and North America (Pfaller et al., 2008 and Lockhart et al., 2012). In this context, we studied the frequency of different Candida species and their antifungal susceptibility profile in ICUs of Zagazig University Hospitals.

In the present study, we observed that NAC was responsible for $(63.59 \%)$ of infection caused by Candida species in patients admitted in ICUs, making it the most common infectious Candida agent. This was consistent with the emergence of predominance of non albicans candidiasis worldwide. Many researchers (Abu-Elteen, 2001; Tortorano et al., 2004 and Bonfietti et $a l ., 2012$ ) have revealed that the distribution of both $C$. albicans and non-albicans was more frequently recovered in ICUs patients compared to other hospital words.

The remarkable increase in the use of invasive devices as central venous catheterization and urinary catheter, advanced age, sever illness as diabetes mellitus and immunosuppression was additional contributors to infection. In addition to improper abuse of antibiotics and start with broad spectrum antibiotics as the first line treatment had been the most important cause of increasing Candida species colonization which causes suppression of the commensal bacterial flora (Sachin et al., 2014). Moreover, admission in ICU itself has come to be an independent risk factor for the enhancement of Candida infection (Zaragoza and Pemán, 2008).

We reported preponderance of Candida isolated from the urine samples (61\%), followed by respiratory tract specimens (16\%), miscellaneous specimens $(15.1 \%)$ and finally $7.9 \%$ isolates from blood samples. Different Candida species were isolated most commonly from urine samples. These results are similar to the findings of many studies who revealed that more than $50 \%$ of Candida isolates from the urine samples belong to NAC species (Zaragoza and Pemán, 2008, Kauffman 2005 and Alvarez-Lerma et al., 2003). Sachin et al., (2014) explained that the NAC spp is well adapted to the urinary tract and also it is difficult to eradicate than $C$. albicans. However, Amer et al., (2015) reported that Candida had been isolated from different samples as follows; $27.54 \%$ from semen, $20.29 \%$ from vagina, $18.84 \%$, from urine, $17.39 \%$ from oral cavity, $11.59 \%$ from stool and $4.35 \%$ from C.S.F.

We found that the prevalence of Candida species was as follows: C. albicans (36.4\%), C. tropicalis (57.7\%), C. glabrata (2.1\%), C. krusei (1.26), C. parapsilosis (1.1\%), C. lusitaniae $(0.6 \%)$, C. kefyr $(0.42 \%)$ and $0.14 \%$ for $C$. dubliniensis, $C$. guilliermondii and $C$. rugosa.

In contrast, the previous study took place in Egypt during 2015. Results showed that the distribution rate of $C$. albicans was the most 
common species representing $68.45 \%$ followed by $16.07 \%$ C. tropicalis, $11.31 \% C$. glabrata and $4.17 \%$ of $C$. krusei represented in patient with candidiasis (Amer et al., 2015). Also, Esmat et al., (2015) revealed that the highest prevalence of Candida isolated from ICU's patients was for $C$. albicans (40.3\%) followed by $C$. tropicalis $(22.2 \%)$; $C$. glabrata (18\%), C. krusei (12.5\%) and $C$. parapsilosis $(4.2 \%)$ were also isolated. The distribution of Candida species was different in the study of Taha et al., (2018) that took place in 2018 and showed decrease in the distribution rate of $C$. albicans to $(36.4 \%)$; but it is still the predominant isolates followed by $25 \%$ for $C$. tropicalis, then $15.9 \%$ for $C$. parapsilosis and $11.4 \%$ for C. krusei. These findings seem compatible with the changes occurred in the Candida infections epidemiology in many countries around the world, which are characterized by a progressive shift from a predominance of $C$. albicans to NAC species.

Furthermore, $C$. albicans still is the most common agent in many researches although its incidence was significant decreasing from 68 to $50 \%$ in Europe and from $64 \%$ to $45 \%$ in Asia (Pfaller et al., 2010 and Pfaller et al., 2013). Moreover, our results were in agreement with a prospective studies of Chakrabarti et al., (2009), who found that the C. tropicalis was the most common (42.1\%) isolated agent and Kaur et al., (2016) who reported that NAC species is predominant (63.3\%) compared by C. albicans (36.7\%) and a lower rate of $C$. glabrata $(10 \%)$ and $C$. parapsilosis $(6.7 \%)$ are isolated while $C$. krusei (3.3\%) and C. kefyr (2.2\%) are relatively rare seen.

The pathogenesis of Candida infection depends on the expression of several virulence factors such as phenotypic switching, adhesions and hydrolytic enzymes. Virulence factors expression may alter according to the infecting species, geographical region, host immune response and type, site and stage of infection. C. tropicalis has its ability to express variation of those virulence factors indicating its ability to contribute to the pathogenesis and produce invasive infections (Yesudhason and Mohanram, 2015).

In this study, the highest resistance rate in all Candida species was against amphotericin B $(11.56 \%)$. However there was no resistance to caspofungin and micafungin (echinocandins class of antifungal) was reported. Low resistance was showed to flucytosine $(2.4 \%)$ among Candida species which similar to the results of Taha et al., (2018) who found thatantibiogram of all Candida species revealed that the pathogens were more sensitive to Flucytosine.

Although triazoles antifungal group was the most frequently used in the treatment of yeast infection, we reported that the resistance rate of C. albicans to voriconazole was $13.46 \%$ and $(8.46 \%)$ to fluconazole. In addition, the resistance rate to voriconazole and fluconazole among NAC species had been reported as $(6.53 \%, 5.1 \%$ respectively).

We observed that, $C$. krusei showed extremely high resistant against fluconazole (100\%) and this was similar to Wissing et al., (2013) results, who reported a high percent of resistance for C. krusei (100\%) to fluconazole because of its intrinsic resistance toward azoles and poor susceptibility to all other antifungals, including amphotericin B.

Our results were supported by of Comert et al., (2007) who reported that C. albicans was more susceptible to fluconazole followed by C. parapsilosis and C. glabrata. However, Ece (2014) revealed that the resistance rate to fluconazole $(27.31 \%)$ was higher than amphotericin B (19.5\%) and flucytosine 
(20\%). In contrast, Kaur et al., (2016) showed high resistance rate towards fluconazole $(37.8 \%)$ compared to amphotericin B (7.8\%).

We did not found any resistance cases of Candida species against echinocandins class of antifungal (caspofungin and micafungin). This result was in agreement with the Dagi et al., (2008) findings. However, resistance of Candida species against echinocandins had been reported in some publications (Wisplinghoff et al., 2014 and Chen et al., 2011).

In conclusion, the study revealed increased incidence of NAC overtime. Our results highlighted the importance of determining the antifungal susceptibility of different Candida species; there was no remarkable resistance for antifungal agents in our hospital. In addition, this study establishes a critical need to antifungal stewardship program to prevent reaching the era of predominant multi-drug resistant strain of candida.

Ethics approval: The study was performed under a protocol approved by the Institutional Review Board of Faculty of Medicine.

\section{References}

Abu-Elteen, K.H. 2001. Increased incidence of vulvovaginal candidiasis caused by Candida glabrata in Jordan. Japan J Infect Dis., 54: 103-107.

Alvarez-Lerma, F., Nolla-Salas, J. and Le'onetal, C. 2003. Candiduria in critically ill patients admitted to intensive care medical units. Intensive Care Medicine., 29(7): 10691076.

Amer, S.M., Elsilk, S.E., Shamma, G.I.T., Abu El-Souod, S.M. and El-Marhomy, A.F. 2015. Epidemiological study on human candidiasis in Tanta hospitals, Egypt. Egypt. J. Exp. Biol., 11(2): 207 - 216.

Anwar, K.P., Malik, A. and Subhan, K.H. 2012. Profile of candidiasis in HIV infected patients. Iran J. Microbiol., 4 (4):204-209.
PMID: 23205253.

Bajwa, S. and Kulshrestha, A. 2013. Fungal infections in intensive care unit: challenges in diagnosis and management. Ann. Med. Health Sci. Res., 3: 238-244.

Bonfietti, L.X., Szeszs, M.W., Chang, M.R., Martins, M.A., Pukinskas, S.R., Nunes, M.O., Pereira, G.H., Aniago, A.M., Purisco, S.U. and Melhem, S.C. 2012. Ten-year study of species distribution and antifungal susceptibilities of Candida bloodstream isolates at a Brazilian tertiary hospital. Mycopathologia. 74: 389-396.

Chakrabarti, A., Chatterjee, S.S., Rao, K.L., Zameer, M.M., Shivaprakash, M.R., Singhi, S., et al., 2009. Recent experience with fungaemia: change in species distribution and azole resistance Scand J. Infect. Dis., 41: 275-284.

Chander, J., Singla, N., Sidhu, S.K. et al., 2013. Epidemiology of Candida blood stream infections: experience of a tertiary care centre in North India. J. Infect. Dev. Ctries., 7: 670-675.

Chen, S., Slavin, M., Nguyen, Q. et al., 2006. Active surveillance for candidemia, Australia. Emerg. Infect. Dis., 12: 15081516.

Chen, S.C., Slavin, M.A. and Sorrell, T.C. 2011. Echinocandin antifungal drugs in fungal infections: a comparison. Drugs. 71:11-41.

Colombo, A.L., Guimaraes, T., Silva, L.R., de Almeida Monfardini, L.P., Cunha, A.K., Rady. P., Alves, T. and Rosas, R.C. 2007. Prospective observational study of candidemia in Sao Paulo, Brazil: incidence rate, epidemiology, and predictors of mortality. Infect. Control Hosp. Epidemiol., 28:570-576.

Comert, F., Kulah, C., Aktas, E., Eroglu, O. and Ozlu, N. 2007. Identification of Candida species isolated from patients in intensive care unit and in vitro susceptibility to fluconazole for a 3-year period. Mycoses. 50(1):52-57.

Dagi, H.T., Findik, D., Senkeles, C. and Arslan, U.2016. Identification and antifungal susceptibility of Candida species isolated from bloodstream infections in Konya, Turkey. Annals of Clinical Microbiology and Antimicrobials. 15:36 DOI 
10.1186/s12941-016-0153-1.

Ece, G.2014. Distribution of yeast-like fungi at a university hospital in Turkey. Jundishapur J. Microbiol., 7(12):e13141.

Esmat, M.M., Mohamed, T. and Abdelrahman, A.H. 2015. Species identification and antifungal susceptibility profile of Candida isolates from ICU patients in Sohag University Hospital, Upper Egypt. Egyptian Journal of Medical Microbiology. 24 (4): 89-97

Goemaere, B., Becker, P., Van Wijngaerden, E., et al., 2018. Increasing candidaemia incidence from 2004 to 2015 with a shift in epidemiology in patients preexposed to antifungals. Mycoses. 61:127-133.

Kauffman, C.A. 2005. Candiduria. Clinical Infectious Diseases. 41 (6): S371-S376.

Kaur, R., Dhakad, M.S., Goyal, R. andKumar, R. 2016. Emergence of non-albicans Candida species and antifungal resistance in intensive care unit patients. Asian Pacific Journal of Tropical Biomedicine. 6(5): 455460.

Lockhart, S.R., Iqbal, N., Cleveland, A.A., Farley, M.M., Harrison, L.H., Bolden, C.B., Baughman, W., Stein, B., Hollick, R., Park, B.J., Chillera, T. 2012. Species identification and antifungal susceptibility testing of Candida bloodstream isolates from population-based surveillance studies in two U.S. cities from 2008 to 2011. J. Clin. Microbiol., 50: 3435-3442.

Oberoi, J.K., Wattal, C., Goel, N., Raveendran, R., Datta, S. and Prasad, K. 2012. Nonalbicans Candida species in blood stream infections in a tertiary care hospital at New Delhi, India. Indian J. Med. Res., 136: $997-$ 1003.

Pappas, P.G., Kauffmanm C.A., Andes, D., Benjamin, D.K., Calandra, T.F., Edwards, J.E., et. al., 2009. Clinical practice guidelines for the management of candidiasis: 2009 update by the Infectious Diseases Society of America. Clin. Infect. Dis., 48: 503-535.

Pfaller, M.A. and Diekema, D.J.2012. Epidemiology of invasive mycoses in North America. Crit. Rev. Microbiol., 36: 1-53.

Pfaller, M.A., Boyken, L., Hollis, R.J., Kroeger, J., Messer, S.A., Tendolkar, S., Diekema,
D.J. 2008. In vitro susceptibility of invasive isolates of Candida spp. to anidulafungin, caspofungin, and micafungin: six years of global surveillance. J. Clin. Microbiol., 46: 150-156.

Pfaller, M.A., Diekema, D.J., Gibbs, D.L., Newell, V.A., Ellis, D., Tullio, V., et al., 2010. Results from the ARTEMIS DISK global antifungal surveillance study, 1997 to 2007: a 10.5 year analysis of susceptibilities of Candida species to fluconazole and voriconazole as determined by CLSI standardized disk diffusion. J. Clin. Microbiol., 48:1366-1377.

Pfaller, M.A., Messer, S.A., Woosley, L.N., Jones, R.N. and Castanheira, M. 2013. Echinocandin and triazole antifungal susceptibility profiles for clinical opportunistic yeast and mold isolates collected from 2010 to 2011: application of new CLSI clinical breakpoints and epidemiological cutoff values for characterization of geographic and temporal trends of antifungal resistance. J. Clin. Microbiol., 51:2571-2581.

Pfaller, M.A. and Diekema, D.J. 2007. Epidemiology of invasive candidiasis: a persistent public health problem. Clin. Microbiol. Rev., 20:133-163.

Sachin, C., Deorukhkar, Saini, S. and Mathew, S. 2014. Non-albicans Candida Infection: An Emerging Threat. Interdisciplinary Perspectives on Infectious Diseases. Article ID 615958, 7 pages.

Sardi, J.C.O., Scorzoni, L., Bernardi, T., FuscoAlmeida, A.M. and Mendes Giannini, M.J.S. 2013. Candida species: current epidemiology, pathogenicity, biofilm formation, natural antifungal products and new therapeutic options. Journal of Medical Microbiology. 62(1):10-24.

Singhi, S. and Deep, A. 2009. Invasive candidiasis in pediatric intensive care units. Indian $\mathrm{J}$. Pediatr., 76(10): 1033-1044.

Taha, M.S.G., Abdel Khalik, H.S., Adds, A.R.G., Abdel Hamid, M.I. and Farahat, A.A.R. 2018. Identification of Candida Species Isolated from Egyptians Patients with Chest Infection Using Integral System Yeast Plus. International Journal of Current Microbiology and Applied Sciences. 7 (1): 
2327- 2335 .

Tortorano, A.M., Peman, J., Bernhardt, H., Klingspor, L., Kibbler, C.C., Faure, O., Biraghi, E., Canton, E., Zimmermann, K., Seaton, S. and Grillot, R. 2004. ECMM Working Group on Candidaemia (2004) Epidemiology of candidaemia in Europe: results of 28-month European confederation of medical mycology (ECMM) hospital based surveillance study. Eur. J. Clin. Microbiol. Infect. Dis. 23: 317-322.

Trick, W.E., Fridkin, S.K., Edwards, J.R., Hajjeh, R.A. and Gaynes Secular, R.P. 2002. Trend of hospital-acquired candidemia among intensive care unit patients in the United States during 1989-1999. Clin. Infect. Dis., 35: 627-630.

Wang, F.J., Zhang, D., Liu, Z.H., Wu, W.X., Ba, H.H. and Dong, H.Y.2016. Species distribution and in vitro antifungal susceptibility of vulvo-vaginal Candida isolates in China. Chin. Med. J. (Engl.), $129,1161-1165$.

Wang, H., Xiao, M., Chen, S.C. et. al. 2010. In vitro susceptibilities of yeast species to fluconazole and voriconazole as determined by the 2010 National China Hospital Invasive Fungal Surveillance Net (CHIFNET) study. J. Clin. Microbiol., 50: 39523959 .

Wisplinghoff, H., Ebbers, J., Geurtz, L., Stefanik, D., Major, Y., Edmond, M.B., et al., 2014.
Nosocomial bloodstream infections due to Candida spp. in the USA: species distribution, clinical features and antifungal susceptibilities. Int. J. Antimicrob. Agents. 43:78-81.

Wissing, H., Ballus, J., Bingold, T.M., Nocea, G., Krobot, K.J., Kaskel, P., et al., 2013. Intensive care unit-related fluconazole use in Spain and Germany: patient characteristics and outcomes of a prospective multicenter longitudinal observational study. Infect. Drug Resist., 6: 15-25.

Xiao, M., Wang, H., Lu, J. et al., 2014. Three clustered cases of candidemia caused by Candida quercitrusa and mycological characteristics of this novel species. J. Clin. Microbiol., 52: 3044-3048.

Yesudhason, B.L. and Mohanram, K. 2015. Candida tropicalis as a Predominant Isolate from Clinical Specimens and its Antifungal Susceptibility Pattern in a Tertiary Care Hospital in Southern India. Journal of clinical and diagnostic research. 9(7): DC14-DC16. doi:10.7860/JCDR/2015/13460.6208.

Zaragoza, R. and Pemán, J. 2008. The diagnostic and therapeutic approach to fungal infections in critical care settings. J. Invasive Fungal Infect. 6 (3): 90- 98.

\section{How to cite this article:}

Ghada E. Amr, Dina M. Atef and Ahmed M. Salah. 2019. Identification and Anti-Fungal Resistance Profile of Different Candida Species Isolated from Patients in ICUs. Int.J.Curr.Microbiol.App.Sci. 8(06): 564-573. doi: https://doi.org/10.20546/ijcmas.2019.806.066 